PROCEEDINGS OF THE $30^{\text {th }}$ European Symposium on Computer Aided Process Engineering (ESCAPE30), May 24-27, 2020, Milano, Italy

(C) 2020 Elsevier B.V. All rights reserved.

\title{
Water Distribution Network Optimization Considering Uncertainties in the Nodes Demands
}

\author{
R. Salcedo-Díaz ${ }^{a^{*}}$, R. Ruiz-Femenia ${ }^{a}$, J. A. Caballero ${ }^{a}$, M. A. S. S. Ravagnani ${ }^{\text {a,b }}$ \\ ${ }^{a}$ Chemical Process Engineering Institute, University of Alicante, Spain \\ ${ }^{b}$ Chemical Engineering Department, State University of Maringá, Brazil \\ raquel.salcedo@ua.es
}

\begin{abstract}
The design of Water Distribution Networks (WDN) can be formulated as an optimization problem for the minimization of the total network cost, which depends on the pipe diameters and the pumping power required. The variability in water demand at nodes can be modelled as a set of finite scenarios generated from a multivariate normal distribution assuming correlations between the selected pair nodes of the network. A disjunctive stochastic Mixed Integer Nonlinear Programming (MINLP) model is proposed for the optimal synthesis of WDN considering correlated uncertainties in nodal demands. Strategies for avoiding nonconvex nonlinearities in the equations are applied to avoid unnecessary complexities. We analyse the effect of different correlation matrices to gather insight into how the model faces uncertainty. A case study was used to test the model and the optimization techniques proposed. Results show that under uncertainty the stochastic solution of the WDN improves the deterministic one (i.e. the design obtained for nominal values of nodes demand), evincing that neglecting uncertainty in the optimization process may lead to suboptimal or, even worse, infeasible design of WDNs.
\end{abstract}

Keywords: Water distribution networks, nodes demand uncertainty, stochastic optimization.

\section{Introduction}

Water Distribution Networks (WDN) are important systems in urban centers and in industrial facilities. The design of WDN can be formulated as an optimization problem, involving, generally, the minimization of the total network cost, which depends on the pipe diameters and flow directions known a priori (Caballero \& Ravagnani, 2019).

In some WDN, there exist variations in the nodal water demands, which could exert a huge influence on the optimal network. In such cases, it is reasonable to account for such uncertainty in the design phase of the WDN. Following this approach, it is guaranteed that the WDN designed under uncertainty is able to cope once the unknow water nodal demands are unveiled, otherwise a design of the WDN assuming nominal values could offer an economically inadequate behaviour. The design under uncertainty one of the key area of Process Systems Engineering, and consequently has been applied to different cases studies (Carrero-Parreño et al., 2019; Ruiz-Femenia et al., 2013). Some authors have considered nodal water demand uncertainty (uncorrelated) in the WDN design, and have used genetic algorithms to find the optimal solution (Branisavljević et al., 2009). Other approach is to handle uncertainty (uncorrelated) through fuzzy logic (Geranmehr et al., 2019). Furthermore, the variability of the water demand (and head) has been represented using log-normal probability distribution uncorrelated functions (Marquez Calvo et al., 2018), but to best our knowledge water demand uncertainty implemented by a set of correlated scenarios with desired marginal distributions for each uncertain 
parameter has not been considered in WDN optimization (for an exhaustive list of works in WDN optimization see Mala-Jetmarova et al, 2018).

\section{Problem statement}

In this work we consider the design of a generic WDN that includes a set of water demand nodes with a set of pipes connecting them (i.e. edges) with fixed flow directions. Given are a set of nodes, described by their elevation and by the expected values and variance of the water flow demand; a set of pipes characterized by their initial and final nodes, their length and whose diameters must be assigned from a set of commercial diameters (with its corresponding price per unit of length); minimum and maximum velocities allowable; and a set of pumps than can provide head at each pipe.

The goal is to determine the configuration of the WDN that minimizes the Total Annualized Cost (TAC) under nodal water demand uncertainty, which is modeled by random variables with the special feature of being correlated. Decisions to be made are of two types: structural and operational. The former includes the diameter and capital cost of each selected pipe, whereas the operational decisions are effective pressure (i.e. total pressure at each node), the velocity, volumetric flow rate and pressure drop at each pipe, and the pump head and its operational cost for each pump.

\section{Representation of the uncertainty}

We model the water demand uncertainty by a set of correlated scenarios, generated by Monte Carlo sampling and with the key feature that each random variable follows the desired marginal distribution (we assume lognormal distribution for the water demand at each node). To enable this flexibility in the scenario modelling, we implement a two-step transformation algorithm (Reyes-Labarta et al., 2014). Given are the expected value and the variance for each nodal water demand, and the correlation matrix. These three inputs define the covariance matrix, whose diagonal elements contain the variances for each variable, while the off-diagonal elements the covariance between variables. First, we apply a standard multivariate normal probability distribution function for each pair of the uncertain parameters to obtain a correlated sampling (Onishi et al., 2017), where each random variable follows a normal marginal distribution (Figure 1a). Then, we apply a transformation to each random variable using the normal cumulative distribution function with the corresponding expected mean and standard deviation to obtain a correlated distribution with uniform marginal distributions on the interval $(0,1)$. And finally, we apply the inverse cumulative distribution of the lognormal distribution (i.e., the desired marginal distribution) to each random variable (Figure 1b). 
Water Distribution Network Optimization Considering Uncertainties in the Nodes Demands
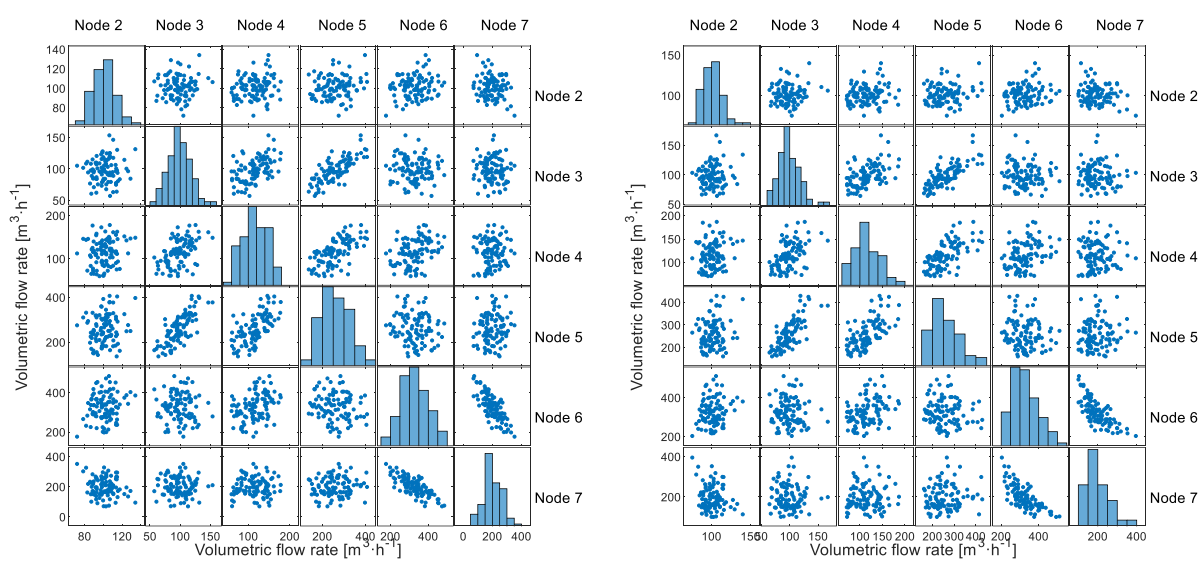

Figure 1. Correlated scenarios between every pairs of nodes for the water demand: a) normal marginal distribution; b) lognormal marginal distributions.

\section{Stochastic mathematical model}

The approach proposed in this work relies on stochastic programming, in particular a twostage stochastic model. In our case, stage-1 decisions, which are taken before the uncertainty is resolved, are given by the design variables, like establishing a new plant or warehouse. In contrast, stage- 2 decisions, which are made after the uncertainty is revealed, model operational variables (mainly production levels and transportation flows) that can be adjusted according to the uncertainty resolution. We assume that the uncertain parameters are described by a set of explicit scenarios with given probability of occurrence. Such scenarios together with their associated probabilities must be provided as input data to the model. In our case, these scenarios are generated from probability distributions using sampling methods.

\subsection{Objective function}

The WDN design is assessed by the Total Annualized Cost (TAC), which includes the annualized pipe capital cost and the pump operation cost. For each scenario $s$, a TAC value is computed, and to collect the performance of the WDN in face of uncertain in a single metric, we minimize the expected value of the TAC, which is compute as follows:

$$
E \text { é } \mathrm{e}^{\mathrm{T}} \mathrm{AC} \underset{\mathrm{u}}{\grave{a}}=\underset{p}{\stackrel{\mathrm{a}}{ }} \operatorname{prob}_{s} T A C_{s}
$$

where $\operatorname{prob}_{s}$ is the given probability of occurrence of scenario $s$.

\subsection{Constraints}

A logic equation is included in the model to assign a diameter for each pipe:

$$
\stackrel{\circ}{a} \mathrm{y}_{i, j, k}=1, " i, j \hat{\mathrm{I}} R_{i, j}
$$

where $R_{i, j}$ define all the pipes by their starting node $i$ and ending node $j$.

In addition, the minimization of the expected value is subject these set of equality and inequality constraints that must be satisfy in each scenario: mass balance at each node; flowrate velocity relation (linearized) at each pipe; mechanical energy balance at each pipe; and the Hazen-Williams at each pipe, that in its linearized form is as follows:. The expression for the last constraint is as follows: 


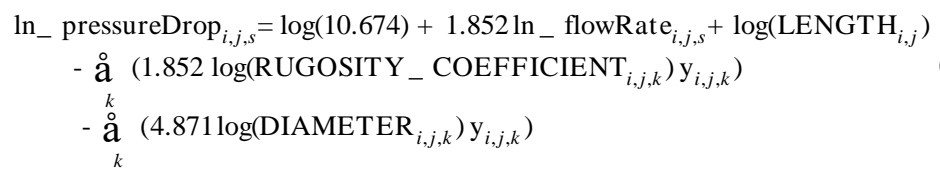

where we use the variable logarithmic transformations of the variables, named $\ln _{-}$pressureDrop $_{i, j, s}$ and $\ln$ flowRate $_{i, j, s}$, instead of their current variables. Also, remark that Eq. (3) arises from a convex hull reformulation of the initial disjunctive model in order to activate only the contribution corresponding to the selected diameter $k$.

\section{Case Study}

The case study is a WDN with seven nodes and eight pipes forming two loops. Figure 2 shows its topology, the node elevations and the direction of the flow. We generate the correlated scenarios with the expected values and variances for water demand shown in Table 2. Node 1 represents the initial water reservoir which flow rate is the summation of water demands in nodes 2 to 7. The correlation matrix elements between two nodes $r$

different from 0: $r_{2,3}=0.6, r_{2,4}=0.8, r_{3,4}=0.7, r_{3,5}=0.4$ and $r_{5,6}=-0.8$.

Table 1. Water demand expected values in nodes.

\begin{tabular}{lrrrrrr}
\hline Water demand $\left(\mathrm{m}^{3} / \mathrm{h}\right)$ & Node 2 & Node 3 & Node 4 & Node 5 & Node 6 & Node 7 \\
\hline Expected value of the & 100 & 100 & 120 & 270 & 330 & 200 \\
Standard deviation & 9.6 & 18.3 & 32.0 & 72.1 & 74.6 & 69.2 \\
\hline
\end{tabular}

All pipes have $1000 \mathrm{~m}$ length and the velocities are bounded between $0.3 \mathrm{~m} / \mathrm{s}$ and $3 \mathrm{~m} / \mathrm{s}$. Pipe diameters are selected from a set of available commercial ones (Table 2). HazenWilliams dimensionless roughness coefficient $\mathrm{C}$ is 130 for all pipes. The use of pumps is considered in case it is necessary to satisfy the nodes demand in any of the scenarios generated. The pumping operational cost is calculated using an electricity cost of 0.24 $\$ / \mathrm{kWh}$

Table 2. Set of available pipe diameters and costs.

\begin{tabular}{lccccccc}
\hline \multirow{2}{*}{ Diameter $(\mathrm{m})$} & 0.0254 & 0.0508 & 0.0762 & 0.1016 & 0.1524 & 0.2032 & 0.254 \\
& 0.3048 & 0.3556 & 0.4064 & 0.4572 & 0.508 & 0.5588 & 0.6096 \\
\hline \multirow{2}{*}{ Cost $(\$ / \mathrm{m})$} & 2 & 5 & 8 & 11 & 16 & 23 & 32 \\
& 50 & 60 & 90 & 130 & 170 & 300 & 550 \\
\hline
\end{tabular}

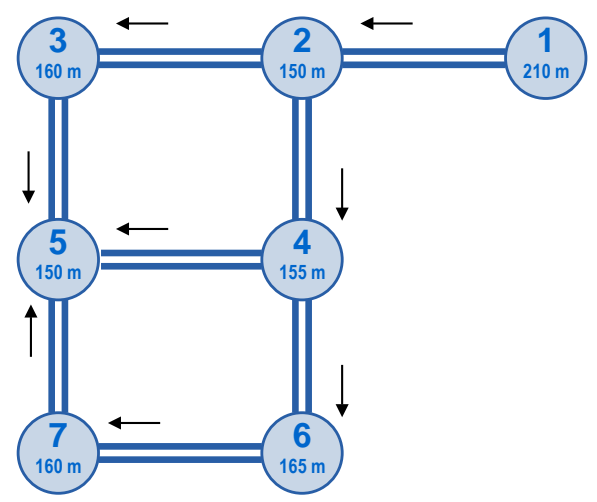

Figure 2. Topology of the WDN studied. 
Water Distribution Network Optimization Considering Uncertainties in the Nodes Demands

\section{Results and discussion}

The stochastic Mixed Integer Nonlinear Programming (MINLP) problem is solved in GAMS using BARON (version 18.11.12). To confirm the suitability of accounting for the uncertainty in the WDN, the problem has been solved again against all the scenarios but fixing the network topology (i.e. binary variables) according to the solution obtained from a deterministic problem, where there is no uncertainty in the nodal water demand (i.e., a unique scenario with water demand equal to the expected value). In addition, the problem has been solved for the case that nodal demands are uncorrelated and correlated according to the above-mentioned correlations coefficients.

Figure 3 shows this comparison in terms of the TAC (\$/y). In both cases the expected TAC for stochastic designs is smaller than the expected TAC for the deterministic ones, which refers to the solution for all scenarios fixing the WDN topology (pipe diameters). Therefore, in some scenarios the system is forced to make use of pumps to satisfy the respective demands of some nodes, thus increasing the cost of the WDN. Another issue with the deterministic solution is that could lead to infeasible solutions as in some scenarios the velocity in pipes must be greater than the imposed upper bound $(3 \mathrm{~m} / \mathrm{s})$ in order to satisfy nodal demands.

Regarding data correlation, both stochastic and deterministic designs yield lower expected TAC when the uncertain parameters are correlated (

Figure 3). Moreover, when distances between nodes are not too large $(1000 \mathrm{~m})$, it is expected a certain correlation between nodes demands. Figure 4 shows the optimal topology (pipe diameters) for the stochastic and deterministic WDN designs with correlated scenarios.

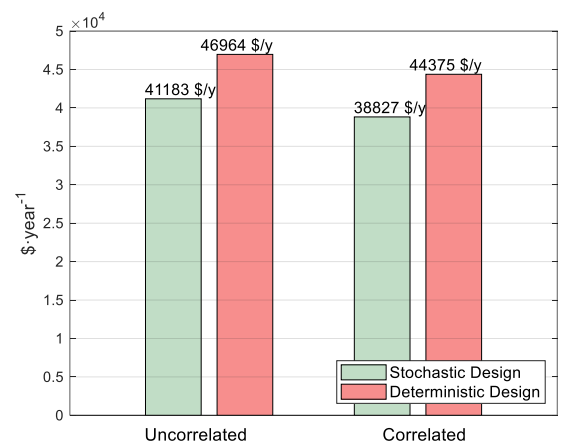

Figure 3. Comparison of the WDN expected total annualized cost between the stochastic and deterministic designs, and for uncorrelated and correlated and nodes demands.

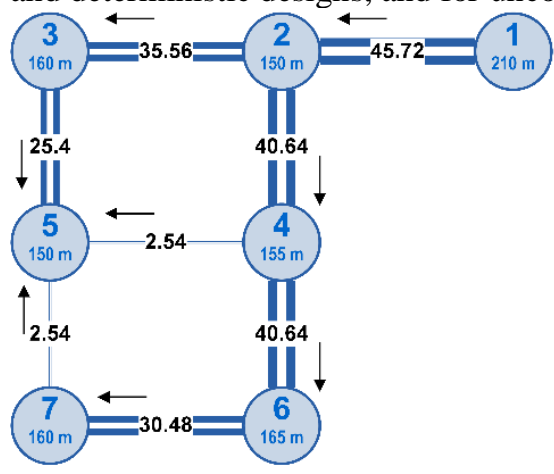

a) Stochastic Design

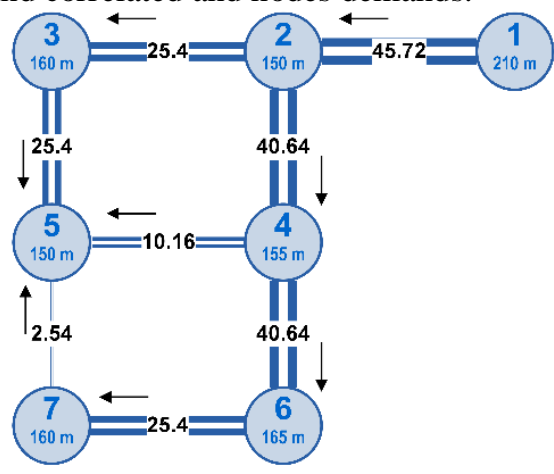

b) Deterministic design 
Figure 4. WDN topology for the stochastic and deterministic designs with scenario correlation (pipe diameters in $\mathrm{cm}$ ).

\section{Conclusions}

A stochastic MINLP optimization model has been developed for the optimal design of water distribution networks under nodal water demand uncertainty. The model has been applied to a case study with 7 nodes seeking to minimize the expected total annualized cost, which includes the pipe capital cost and the pumping operational cost. The solution of the stochastic problem is compared with that obtained fixing the pipe diameters according to the solution of the problem that assumes no variability in water demand at nodes. The results show that the stochastic design reduces the expected TAC by more than $12 \%$ with respect to the deterministic one. This is mainly due to the need of using pumps to satisfy the nodes demand in some scenarios in the deterministic design. Additionally, the deterministic design could become infeasible due to the excessive water velocity in pipes needed to satisfy the water demand in some scenarios. These facts demonstrate that neglecting uncertainty in the optimization process may lead to suboptimal or, even worse, infeasible design of WDNs.

\section{Acknowledgements}

The authors acknowledge financial support to the Spanish «Ministerio de Economía, Industria y Competitividad» under project CTQ2016-77968-C3-2-P (AEI/FEDER, UE).

\section{References}

Branisavljević, N., Prodanović, D., \& Ivetić, M. (2009). Uncertainty reduction in water distribution network modelling using system inflow data. Urban Water Journal, 6, 69-79.

Caballero, J. A., \& Ravagnani, M. A. S. S. (2019). Water distribution networks optimization considering unknown flow directions and pipe diameters. Computers \& Chemical Engineering, 127, 41-48.

Carrero-Parreño, A., Quirante, N., Ruiz-Femenia, R., Reyes-Labarta, J. A., Salcedo-Díaz, R., Grossmann, I. E., \& Caballero, J. A. (2019). Economic and environmental strategic water management in the shale gas industry: Application of cooperative game theory. AIChE Journal, 65, e16725.

Geranmehr, M., Asghari, K., \& Chamani, M. R. (2019). Uncertainty analysis of water distribution networks using type-2 fuzzy sets and parallel genetic algorithm. Urban Water Journal, 16, 193-204.

Mala-Jetmarova, H., Sultanova, N., \& Savic, D. (2018). Lost in optimisation of water distribution systems? A literature review of system design. Water (Switzerland), 10.

Marquez Calvo, O. O., Quintiliani, C., Alfonso, L., Di Cristo, C., Leopardi, A., Solomatine, D., \& de Marinis, G. (2018). Robust optimization of valve management to improve water quality in WDNs under demand uncertainty. Urban Water Journal, 15, 943-952.

Onishi, V. C., Ruiz-Femenia, R., Salcedo-Díaz, R., Carrero-Parreño, A., Reyes-Labarta, J. A., \& Caballero., J. A. (2017). Optimal Shale Gas Flowback Water Desalination under Correlated Data Uncertainty. Computer Aided Chemical Engineering, 40, 943 - 948.

Reyes-Labarta, J. A., Salcedo-Díaz, R., Ruiz-Femenia, R., Guillén-Gosálbez, G., \& Caballero, J. A. (2014). Handling of uncertainty in life cycle inventory by correlated multivariate lognormal distributions: Application to the design of supply chain networks. Computer Aided Chemical Engineering, 33, 1075-1080.

Ruiz-Femenia, R., Guillén-Gosálbez, G., Jiménez, L., \& Caballero, J. A. (2013). Multi-objective optimization of environmentally conscious chemical supply chains under demand uncertainty. Chemical Engineering Science, 95, 1-11. 